\title{
Glottopol
}

Revue de sociolinguistique en ligne

$34 \mid 2020$

Les « langues de France ", 20 ans après

\section{Les langues régionales au Parlement, ou l'éternel retour}

Philippe Martel et Marie-Jeanne Verny

\section{(2) OpenEdition}

Journals

Édition électronique

URL : https://journals.openedition.org/glottopol/375

DOI : $10.4000 /$ glottopol.375

ISSN : 1769-7425

Éditeur

Presses universitaires de Rouen et du Havre

Référence électronique

Philippe Martel et Marie-Jeanne Verny, "Les langues régionales au Parlement, ou l'éternel retour », Glottopol [En ligne], 34 | 2020, mis en ligne le 01 juillet 2020, consulté le 02 octobre 2021. URL : http:// journals.openedition.org/glottopol/375; DOI : https://doi.org/10.4000/glottopol.375

Glottopol 


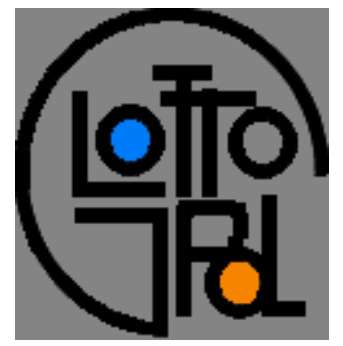

\section{GLOTTOPOL}

Revue de sociolinguistique en ligne $\mathrm{n}^{\circ} 34$ - juillet 2020

Les "langues de France " : 20 ans après

\section{SOMMAIRE}

Hommages à Jean Le Dî

Christian Lagarde : "Langues de France ». Au-delà du symbolique?

Entretien avec Bernard Cerquiglini, par Christian Lagarde.

Entretien avec Paul de Sinety, Délégué général à la langue française et aux langues de France, par Christian Lagarde.

Georg Kremnitz : La problématique initiale de la liste Cerquiglini et ses effets ultérieurs.

Alain Viaut : De "langue régionale » à "langue de France » ou les ombres du territoire.

Wanda Mastor: Le statut constitutionnel des langues régionales en droit comparé. De la reconnaissance à l'indifférence.

Philippe Martel, Marie-Jeanne Verny: Les langues régionales au Parlement, ou l'éternel retour.

Romain Colonna : Les «langues de France »: des langues non-étatiques au pays de l'Étatnation.

Hervé le Bihan : La langue bretonne : une visibilité toute en retenue.

Véronique Bertile : Les langues d'outre-mer : des langues de France? Approche juridique.

Jacques Vernaudon: Les langues polynésiennes et kanak, des "langues de France» en contexte de décolonisation.

Luc Biichlé : Qu'advient-il de l'arabe de France? Mise en perspective sociolinguistique...

Pascal Ottavi : L'épervier, la cage et le passereau.

Marielle Rispail : Le francique lorrain, langue de France? Réflexions et témoignages.

\section{Compte rendu de lecture}

Par Salih Akin : Jean Le Dû \& Yves Le Berre, Métamorphoses. Trente ans de sociolinguistique à Brest (1984-2014), Brest, Centre de Recherche Bretonne, 2019, 302 p.

http://glottopol.univ-rouen.fr 


\title{
LES LANGUES RÉGIONALES AU PARLEMENT, OU L'ÉTERNEL RETOUR
}

\author{
Philippe Martel, Marie-Jeanne Verny \\ Université Paul Valéry, Montpellier, laboratoire LLACS
}

Sur la base du corpus de milliers de pages qui leur a servi de base (qu'ils aimeraient voir publié un jour dans un document unique), à savoir les débats au Sénat et à l'Assemblée Nationale à l'occasion de telle ou telle proposition de loi, les auteurs de cet article sont amenés à dresser le constat qu' on n'y traite presque exclusivement que des « langues régionales » de France et jamais des "langues de France », notion introduite par le Rapport Cerquiglini de 1999. Au-delà des langues de l'hexagone et de la Corse, on débat rarement en effet des langues d'Outre-mer qu'englobe aussi cette notion, et aucune mention n'est faite des langues dites « minoritaires sans territoire » (berbère, arabe dialectal, yiddish, romani, arménien occidental, judéo-espagnol, langue des signes française) prises également en compte par la DGLFLF. Ces lacunes sont sans doute le signe d'une boucle discursive : le législateur ne saurait traiter d'un objet qui n'est pas inscrit dans la loi, ni - sauf à la marge d'articles spécifiques à certains territoires - dans la Constitution.

Les auteurs n'ont pas la prétention de livrer ici une réflexion de caractère strictement sociolinguistique. Ils proposent plutôt, modestement, de livrer à la recherche sociolinguistique la présentation organisée d'un fonds documentaire d'archives important, éparpillé, jamais encore inventorié ni édité, encore moins étudié en tant que tel : que mettre, en effet à ce jour, dans une bibliographie qui demeure à nourrir et à construire, si ce n'est les références aux textes juridiques et aux débats eux-mêmes ? Mais, comme on pourra en juger, ce corpus, en tant que pan d'une histoire et d'une sociologie des mentalités, s'avère très révélateur, en termes de politique linguistique, et de politique tout court. Nous ne chercherons pas ici à faire l'histoire chronologique de ces débats, d'autant plus que, somme toute, ils n'ont donné lieu à aucune traduction concrète dans le corpus législatif ou constitutionnel, si on excepte l'introduction en 2008 de l'article 75-1 dans la Constitution; mais plutôt à en dégager les lignes de force et la nature des principaux arguments échangés (assez stables d'un débat à l'autre) au cours de discussions portant aussi bien sur les textes proposés que sur les amendements déposés par les élus de tous bords. Cet examen - illustré d'abondantes citations plus ou moins longues où il est possible de voir se développer les argumentaires et le débat contradictoire - devrait nous permettre de dresser le tableau des positions des partis politiques - à la vérité eux-mêmes la plupart du temps partagés - face à la question des langues régionales.

C'est un lieu commun de dire que depuis la loi Deixonne de 1951, la question des langues régionales est revenue régulièrement devant le parlement (Assemblée et Sénat), sans être jamais réglée. En fait, c'est par dizaines que l'on compte les propositions de loi déposées par des élus 
représentant pratiquement tous les partis, et jamais discutées. Tout au plus l'enseignement de ces langues est-il évoqué dans les textes généraux qui ont réformé l'Éducation Nationale depuis la loi Haby de $1975^{1}$ jusqu'à la loi Peillon de refondation de l'école en $2013^{2}$.

Mais depuis un peu plus de dix ans, le nombre des débats publics a sensiblement augmenté, débouchant sur la constitution d'un corpus de discours qui permet de prendre la température du monde politique sur cette question.

Il y a eu les débats sur la Charte européenne des langues régionales, notamment en $2008^{3}$, quand le sujet est furtivement inséré dans une discussion plus générale sur la modernisation de la vie politique, mais avec un résultat, l'article 75-1: « Les langues régionales appartiennent au patrimoine de la France », et, de façon plus explicite en 2014 et 2015, sans résultat, cette fois 4 .

Il y a eu par ailleurs plusieurs propositions de loi (on note d'emblée qu'il n'y a jamais eu de projet de loi déposé par quelque gouvernement que ce soit...) sur la signalétique, sur l'enseignement, ou sur le statut des langues régionales en général : la proposition du sénateur socialiste Roland Courteau en $2010^{5}$, celle du sénateur, socialiste lui aussi Robert Navarro en $2011^{6}$, celle du député écologiste Paul Molac en 2015 à l'Assemblée ${ }^{7}$, celle déposée au Sénat par Philippe Bas (Les Républicains) en octobre $2015^{8}$, celle enfin du socialiste Bruno Le Roux en $2016^{9}$. Au moment où nous écrivons, Paul Molac dépose, sous une nouvelle étiquette, une nouvelle mouture de sa proposition de loi $^{10}$, dont l'avenir semble d'ailleurs d'ores et déjà compromis, puisque le texte a été vidé de la plupart de ses articles avant d'être adopté par l'Assemblée le 13 février 2020 en première lecture.

À l'exception de la Proposition de Loi Bas de 2015, devenue caduque avant d'être examinée, tous les textes évoqués ont fait l'objet de débats en commission, et de débats plus ou moins longs en séance plénière (dans le cadre souvent d'une niche parlementaire). Certains de ces débats, notamment ceux portant sur la Charte, ont rencontré un certain écho dans l'opinion, qu'il s'agisse de la presse ou des réseaux sociaux, mais nous laisserons de côté ces échos pour nous concentrer sur les débats proprement dits au Sénat et à l'Assemblée, tels que nous les révèlent les comptes rendus officiels des deux assemblées, disponibles sur leurs sites respectifs.

Ces débats impliquent un certain nombre d'intervenants (en fonction du nombre des élus présents en séance) : rapporteurs, ministres concernés, quand ils assistent à la discussion, porteparole des groupes, et députés s'exprimant à titre individuel, qu'ils soient ou non membres des commissions concernées (Lois, Affaires culturelles pour l'essentiel). D'un débat à l'autre reviennent donc des « habitués », voire des « spécialistes », rarement de vrais ténors. Il convient d'essayer de décoder les discours construits qu'ils produisent, mais aussi ce que révèlent les interruptions au fil des discussions, conformes à un usage aussi vieux que le parlementarisme.

\footnotetext{
1 Article 12 de la loi Loi $\mathrm{n}^{\circ} 75-620$ du 11 juillet 1975 relative à l'éducation : https://www.legifrance.gouv.fr/affichTexte.do?cidTexte=JORFTEXT000000334174

${ }^{2}$ Article 40 de la loi Loi n`2013-595 du 8 juillet 2013 d'orientation et de programmation pour la refondation de l'École de la République : https://www.education.gouv.fr/cid102387/loi-n-2013-595-du-8-juillet-2013-dorientation-et-de-programmation-pour-la-refondation-de-l-ecole-de-la-republique.html

${ }^{3}$ Voir le contenu des débats au Sénat : https://www.senat.fr/seances/s200805/s20080513/s20080513002.html et à l'Assemblée nationale : http://www.assemblee-nationale.fr/13/cri/2007-2008-extra/20081013.asp (après l'article 30 sexties).

${ }^{4}$ Voir les débats au Sénat: https://www.senat.fr/dossier-legislatif/pj114-662.html et le dossier sur le site de l'Assemblée nationale :

nationale.fr/dyn/14/dossiers/alt/charte europeenne langues regionales

http://www.assemblee-

${ }^{5}$ https://www.senat.fr/rap/110-293/110-2930.html

${ }^{6} \mathrm{http}: / /$ www.senat.fr/rap/110-657/110-6570.html

${ }^{7} \mathrm{http}: / /$ www.assemblee-nationale.fr/14/propositions/pion3288.asp

${ }^{8} \mathrm{http}: / /$ www.senat.fr/dossier-legislatif/ppl15-096.html

${ }^{9} \mathrm{http}: / /$ www.assemblee-nationale.fr/14/propositions/pion4096.asp

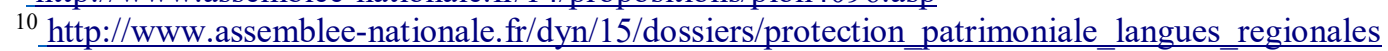


La question des langues régionales telle qu'elle est abordée au parlement, on le verra, est certes un problème "technique », destiné à savoir si elles ont une place et laquelle dans le champ juridique, mais elle est avant tout une question profondément idéologique, ancrée sur des représentations sociopolitiques, socioculturelles, et au bout du compte fondamentalement sociolinguistiques, qui ont la vie dure, et dont nous avons tenté de dresser la cartographie thématique.

\section{La question des langues régionales, un problème juridique ?}

\section{Un problème « technique » : la ratification de la Charte européenne}

La première dimension des débats sur le sujet est la dimension technique, bien évidemment, puisqu'il est question de la Constitution et de la législation. En ce qui concerne la Constitution, le débat porte essentiellement sur la possibilité pour la France de ratifier la Charte européenne des langues régionales et minoritaires, adoptée en 1992 par le Conseil de l'Europe, signée en 1999 sous le gouvernement Jospin, mais jugée par le Conseil constitutionnel comme par le Conseil d'État incompatible avec la Constitution. Pour ces deux instances, la Charte contredit l'article 1, qui définit la France comme une République Une et indivisible et garantit l'égalité entre tous les citoyens. Elle contredit aussi l'article 2, tel que modifié en 1992 au profit de la désignation du français comme langue de la République.

En 2008, la question des langues régionales s'invite dans deux débats dans lesquels elle n'apparaissait pas au départ (ce qui, entre les deux, débouche sur un débat spécifiquement consacré à ces langues, mais sans vote...).

C'est le cas d'abord en janvier ${ }^{11}$, dans le cadre d'un débat consacré à un autre problème, celui de savoir si le traité de Lisbonne doit être ou non soumis à référendum. À l'Assemblée, la question des langues régionales est posée par quelques amendements, venus notamment des bancs socialistes ${ }^{12}$, mais aussi par une proposition de loi émanant de Victorin Lurel et 118 de ses collègues du groupe socialiste, radical, citoyen et divers gauche et apparentés ${ }^{13}$.

Un autre amendement est déposé par l'UMP, invitant à joindre une mention les concernant dans l'article 2 de la Constitution ${ }^{14}$, puis au Sénat, sous la forme d'un amendement socialiste

\footnotetext{
${ }^{11}$ On trouvera les débats à l'Assemblée sur ce lien : http://www.assemblee-nationale.fr/13/cra/2007-2008/099.asp, https://www.senat.fr/seances/s200801/s20080129/s20080129013.html et au Sénat pages http://www2.senat.fr/seances/s200801/s20080129/s20080129006.html et suivantes.

12 Article additionnel avant l'article $1^{\mathrm{er}}$. Présentation par la présidente de séance : l'amendement $\mathrm{n}^{\circ} 5$ rectifié bis, [...] est ainsi libellé : Avant l'article 1 ${ }^{\mathrm{er}}$, ajouter un article additionnel ainsi rédigé : Après l'article 53-2 de la Constitution, il est inséré un article ainsi rédigé : «Art. ... - Dans le respect du premier alinéa de l'article 2, la République française peut ratifier la Charte européenne des langues régionales ou minoritaires du Conseil de l'Europe. »)

${ }^{13}$ Proposition de loi constitutionnelle Victorin Lurel et alii tendant à la reconnaissance des langues régionales : http://www.assemblee-nationale.fr/13/propositions/pion0657.asp (20 février 2008).

14 http://www.assemblee-nationale.fr/13/amendements/0561/056100013.asp, Marc Le Fur et alii, AVANT L'ARTICLE PREMIER, insérer l'article suivant: Après l'article 53-2 de la Constitution, il est inséré un article 53-3 ainsi rédigé : «Art. 53-3. - La République française peut adhérer à la Charte européenne des langues régionales ou minoritaires, signée le 7 mai $1999 »$
} 
proposant, lui, un article 53-2 de la même Constitution ${ }^{15}$ complété par l'autorisation donnée à la France de ratifier la Charte ${ }^{16}$.

On note au passage que la solution consistant à enrichir l'article 2 par une mention associant français et langues régionales avait déjà été proposée en 1992, mais qu'elle avait été écartée par le gouvernement de l'époque au motif qu'il s'agissait de garantir le statut du français face à l'anglais dans le cadre de l'Europe post-Maastricht, mais que les langues régionales n'étaient nullement menacées. On a vu depuis de quoi il retournait dans le monde réel, aussi bien pour ce qui concerne l'emprise de l'anglais (peu menacé, lui) que pour ce qui concerne les langues régionales.

De toute façon, le débat de janvier 2008 débouche sur un refus du gouvernement de revenir sur l'article 2, refus à peine compensé par sa promesse de traiter du problème dans une révision constitutionnelle à venir.

Le 7 mai suivant, la ministre de la culture Christine Albanel lit devant l'Assemblée une déclaration du gouvernement ${ }^{17} \mathrm{~d}$ 'où il ressort que, même s'il n'est pas question de modifier la Constitution, il y aura bel et bien un projet de loi gouvernemental sur le sujet. À l'époque, les députés, et peut-être même Christine Albanel elle-même, ignorent que ce projet ne verra jamais le jour. On note par ailleurs que si cette déclaration est suivie d'un débat qui mobilise plusieurs élus, aucun vote, on l'a dit, ne le conclut, ce qui lui enlève une bonne partie de sa portée potentielle, quel que soit l'intérêt indéniable des propos échangés. La question ressurgit quelques semaines plus tard (le 22 mai) dans le débat constitutionnel dit de «modernisation de la vie politique $\gg^{18}$. L'opposition remonte à l'assaut, toujours en proposant de compléter l'article 2 en associant français langue commune, et respect des langues régionales. Ainsi en est-il de Noël Mamère, alors député des Verts :

Face au refus d'inscrire toute référence à la Charte européenne des langues régionales et minoritaires dans notre Constitution, notre amendement 304 vise à compléter le premier alinéa de l'article 2 de la norme suprême par la mention suivante : « Les langues régionales de France sont également reconnues par la République ». L'on sortirait ainsi du statut de simple tolérance de ces langues pour arriver à une reconnaissance a minima. Ce ne serait certes qu'un petit progrès, mais il serait d'autant mieux venu que les aides aux écoles dispensant les enseignements afférents sont très faibles, cependant que des études montrent que l'apprentissage précoce d'une langue régionale aide à bien maitriser le français. Je n'ignore pas que le rapporteur va présenter un amendement à ce sujet mais sa rédaction est très différente.

On note la réponse du rapporteur UMP Jean-Luc Warsmann :

Dans son souci de répondre de manière constructive aux attentes des parlementaires, la commission des lois a en effet souhaité réagir aux amendements relatifs aux langues régionales déposés par plusieurs collègues, dont MM. Mamère et Folliot. Il ne s'agit pas de remettre en cause l'article 2 de la Constitution, mais de donner un ancrage constitutionnel aux langues régionales, comme beaucoup en ont fait le vœu lors du débat organisé ici-même le 7 mai dernier.

La discussion débouche, non sans mal, sur l'adoption d'un amendement (le $\left.n^{\circ} 605\right)$ de Warsmann, introduisant la référence aux langues régionales dans l'article 1 (« les langues

\footnotetext{
15 Voir https://www.senat.fr/cra/s20080129/s20080129 8.html : Amendement n5, présenté par MM. Marc (et alii) : « Avant l'article $1^{\text {er }}$, ajouter un article additionnel ainsi rédigé : Après l'article 53-2 de la Constitution, il est inséré un article ainsi rédigé : «Art. ... - Dans le respect du premier alinéa de l'article 2, la République française peut ratifier la Charte européenne des langues régionales ou minoritaires du Conseil de l'Europe. ».

${ }^{16}$ Voir sur le site du Sénat « Projet de loi constitutionnelle de modernisation des institutions de la Ve République » http://www.senat.fr/rap/107-387/107-38727.html.

${ }^{17} \mathrm{http}: / /$ www.assemblee-nationale.fr/13/cra/2007-2008/153.asp

${ }^{18} \mathrm{http}$ ://www.assemblee-nationale.fr/13/cra/2007-2008/165.asp.
} 
régionales appartiennent à son patrimoine » [de la France]. Son argumentation : insérer les langues régionales dans l'article 2 à la suite du français aboutirait à affaiblir la force de l'affirmation de son statut de langue de la République. Les insérer dans l'article 1, à la suite de la phrase définissant la France comme République décentralisée, les rangerait au rang des symboles de la Nation.

Le Sénat, de son côté, saisi du texte en juin 2008, élimine sèchement toute mention des langues régionales ${ }^{19}$.

C'est en seconde lecture que celles-ci sont enfin intégrées à la Constitution, mais sous la forme d'un article 75-1. Non sans quelque paradoxe, ce qui est défini comme «patrimoine national » est donc de fait, relégué dans la partie de la Constitution concernant les collectivités locales. Un amendement de suppression du sénateur communiste Yvan Renar déposé au Sénat au cours de la seconde lecture le 16 juillet ${ }^{20}$ souligne du coup le danger de voir cet article servir de prétexte pour « organiser un transfert de charges en direction des collectivités territoriales ».

L'article 75-1 n'en est pas moins adopté par le congrès le 21 juillet, avec l'ensemble du texte révisant la Constitution, par une majorité de 539 voix, soit une de plus que la majorité requise des trois cinquièmes ${ }^{21}$.

Ceci étant, une décision du Conseil constitutionnel, dont la vigilance sur la question n'est plus à démontrer, expliquera en mai 2011 que l'article 75-1 ne garantit aucun droit spécifique à qui que ce soit, puisque la Constitution garantit déjà, de toute façon, la liberté d'expression, y compris en langue régionale, donc. On se trouve là somme toute face à un article constitutionnel qui ne sert à rien ${ }^{22}$.

Le débat reprend en $2014^{23}$ et 2015 , sous une autre législature, et en conséquence d'une promesse de ratification de la Charte formulée par François Hollande au cours de sa campagne électorale (la proposition $\mathrm{n}^{\circ} 56$ de son programme).

L'Assemblée insère le 28 janvier 2014 dans la Constitution un article 53-3 autorisant la France à ratifier la Charte, adopté par 361 voix contre 149 (la droite pour l'essentiel) ${ }^{24}$. L'argument des opposants : il s'agit d'une proposition de loi constitutionnelle, non d'un projet soumis par le gouvernement. De ce fait, soit on s'en tient là et dans ce cas, aux termes de la Constitution, il faut un référendum, soit le gouvernement, à l'issue des débats et en fonction de leur résultat, décide, ou non, de soumettre un projet de loi reprenant les termes de la proposition, et dans ce cas, on ne voit pas l'utilité de ladite proposition, sinon comme « tour de chauffe » ou «test », pour reprendre les termes employés dans la discussion. À cette date, le Sénat étant contrôlé par la gauche, il aurait été possible effectivement de faire voter le congrès sur un projet de loi et son adoption par les trois-cinquièmes des parlementaires n'aurait donc pas été impossible.

Mais c'est seulement le 31 juillet 2015 que le gouvernement propose un projet de loi constitutionnelle sur le sujet ${ }^{25}$. Lorsque ce projet arrive le 27 octobre 2015 devant le Sénat, dans lequel la droite vient de retrouver sa majorité habituelle, le vote (179 voix contre 155) aboutit à un refus d'examiner le texte ${ }^{26}:$ il n'y aura donc pas de navette avec l'Assemblée, et le projet se retrouve enterré avant même d'avoir été réellement discuté. C'est pour l'essentiel au nom du

\footnotetext{
${ }^{19} \mathrm{http}: / /$ www.senat.fr/cra/s20080624/b s20080624 34.html

${ }^{20} \mathrm{https}: / /$ www.senat.fr/seances/s200807/s20080715/s20080715004.html

${ }^{21} \mathrm{https}: / /$ www.senat.fr/seances/s200807/s20080721/s20080721_mono.html

${ }^{22} \mathrm{https}: / / \mathrm{www} . c 0 n s e i l-c o n s t i t u t i o n n e l . f r / d e c i s i o n / 2011 / 2011130 \overline{Q P C} . \mathrm{htm}$

23 Rapport Urvoas visant à ratifier la Charte européenne des langues régionales ou minoritaires http://www.assemblee-nationale.fr/14/rapports/r1703.asp (14 janvier 2014)

${ }_{24}$ http://www.assemblee-nationale.fr/14/ta/ta0283.asp - voir le compte rendu des débats à l'adresse http://www.assemblee-nationale.fr/14/cri/2013-2014/20140146.asp\#P178332

${ }^{25} \mathrm{https}: / / \mathrm{www}$. senat.fr/leg/pjl14-662.html

26 https://www.senat.fr/espace presse/actualites/201510/quel avenir pour le projet de loi constitutionnel autorisant la ratification_de la charte europeenne des langues regionales_ou minoritaires.html
} 
droit constitutionnel et de son rapport au droit international que ce refus de délibérer est justifié. En gros, on a affaire à une double incompatibilité juridique :

- la Charte est en contradiction avec la Constitution dans ses articles 1 et 2, et la déclaration interprétative dont la France l'a accompagnée en 1999 pour se couvrir sur ce front ne garantit absolument pas contre la possibilité qu'un gouvernement aille un jour au-delà des 39 dispositions acceptées par la France (et qui, elles, sont compatibles avec la Constitution, de l'aveu même du Conseil constitutionnel, comme tous les intervenants ne manquent pas de le rappeler avant de passer outre quand ils sont contre la Charte) pour autoriser la coofficialité des langues régionales et du français, et reconnaitre des droits spécifiques à la communauté de leurs locuteurs.

- d'autre part, la Charte du Conseil de l'Europe interdisant toute réserve, la déclaration interprétative n'a aucune valeur au regard du droit international.

Bref, échec et mat, même si on note au passage que cette austère argumentation juridique est absente du débat précédent en 2008. Et du coup, une législature plus tard et compte tenu des rapports de force dans les deux assemblées, il semble peu probable que qui que ce soit ait l'idée dans un avenir envisageable de relancer le débat constitutionnel, que ce soit sur la Charte ou sur l'article 2.

\section{À l'ombre de la Constitution}

Ce alors même que cet article 2 peut aussi être convoqué dans les débats sur les propositions de loi concernant le statut des langues régionales, signe que le débat constitutionnel et le débat législatif sont difficilement séparables, quoi qu'en disent certains acteurs du débat qui pensent qu'une loi pourrait passer sans censure constitutionnelle.

Nous avons cité plus haut ces propositions.

La première question que l'on peut se poser à leur propos est celle de leur taille.

Deux solutions possibles :

- soit une proposition détaillée, comportant plusieurs dizaines d'articles. C'est le cas de la proposition de loi Navarro devant le Sénat $(58 \text { articles })^{27}$ ou de la proposition Jung à l'Assemblée (69 articles) ${ }^{28}$.

- D'autres propositions (Molac par exemple ${ }^{29}$ ) se limitent à une dizaine d'articles, voire moins.

Deux visions ici s'affrontent :

- Soit l'on considère qu'une loi-cadre, précisant quelques grands principes, suffit, et qu'il revient au gouvernement de la mettre en application par décrets.

- Soit l'on considère, instruit par l'expérience de la loi Deixonne, et par d'autres lois plus récentes portant sur toutes sortes de sujets, que le gouvernement peut parfaitement se dispenser de prendre de tels décrets, condamnant du même coup la loi à l'inefficacité totale. Dès lors, la loi doit être la plus détaillée possible.

L'histoire s'est chargée de mettre d'accord ces deux visions, puisqu'aucun des textes déposés n'a été définitivement adopté (et dans le cas de la proposition Navarro, faute de temps, le débat s'est arrêté avant même la discussion de l'article 1). Mais cela n'enlève rien à l'importance du débat.

Pour en revenir à la question de l'incidence de la Constitution sur les propositions de loi concernant les langues régionales, l'examen des débats révèle l'importance parfois déterminante de cette incidence.

\footnotetext{
${ }^{27} \mathrm{https}: / /$ www.senat.fr/leg/ppl10-251.html

${ }^{28} \mathrm{http}: / /$ www.assemblee-nationale.fr/13/propositions/pion3008.asp

${ }^{29} \mathrm{http} / / / \mathrm{www}$.assemblee-nationale. fr/14/propositions/pion3288.asp
} 
La rapporteure de la proposition Navarro, la sénatrice (UMP) Colette Melot $^{30}$, invoque (entre autres) l'article 2, avant de conclure à un avis défavorable au nom de la commission des affaires culturelles.

Et le ministre en exercice, Luc Chatel (UMP lui aussi), oppose le même avis défavorable, en s'appuyant là encore, entre autres arguments, dans une (très) longue intervention, sur l'article 2, en fonction duquel «il ne peut être reconnu aucun droit à pratiquer une langue autre que le français dans la sphère de la vie publique, que ce soit la justice, les services publics ou l'enseignement » (séance du 30 juin 2011 au Sénat ${ }^{31}$ ). On note qu'appliquée dans toute sa rigueur, une telle position pourrait mener tout simplement à l'interdiction de toute forme d'enseignement d'une langue régionale à l'école, publique ou non.

À l'Assemblée en mars 2013, la discussion d'un amendement 27-bis à la loi dite de refondation de l'école concernant les langues régionales ${ }^{32}$ amène le ministre (socialiste, lui) Vincent Peillon à recourir lui aussi à l'article 2 pour souligner que l'enseignement de ces langues ne peut être que facultatif, non obligatoire :

De ce point de vue, la rédaction de la commission est, après expertise, frappée d'inconstitutionnalité pour les raisons qui ont été évoquées. Aux termes de l'article 2 de notre Constitution « La langue de la République est le français », ce qui signifie que les enseignements en langue régionale que nous favorisons ne peuvent être que facultatifs et requièrent, je le répète, l'accord des personnes responsables des élèves mineurs ou des élèves majeurs.

Du coup, la majorité socialiste adopte un amendement du rapporteur subordonnant toute utilisation de la langue et de la culture locale à une autorisation explicite des parents d'élèves qui, appliquée et interprétée par des décideurs de terrain malveillants, comme il en existe un certain nombre, aurait pu rendre tout bonnement impossible toute véritable utilisation des langues régionales ailleurs que dans le cadre étroit des quelques cours spécifiquement consacrés à la langue (par exemple en français ou en histoire-géographie).

Il faudra une forte mobilisation des associations de défense des langues concernées pour amener le Sénat, puis l'Assemblée en seconde lecture, à adopter une rédaction moins problématique pour un article qui au final inscrit la possibilité d'un enseignement bilingue dans la loi :

M. Vincent Peillon, ministre. [...] Je tiens tout d'abord à préciser que la précédente version de l'article 27 bis ne marquait nullement une régression, puisqu'elle conservait tous les apports intégrés jusque-là et que j'avais déjà pu prodiguer mes encouragements au développement concret de ces langues. J'ai aussi pu constater que des motifs sans doute un peu politiciens conduisaient certains à vouloir améliorer le texte. Mais il faut s'en réjouir. L'article 27 bis, dans sa nouvelle rédaction, présente des avancées jusque-là inconnues. Premièrement, l'enseignement des langues régionales sera favorisé, en particulier dans les régions où elles sont le plus en usage. Deuxièmement, l'enseignement bilingue - vous l'avez fort bien dit, monsieur Molac - est introduit dans la loi. C'est la première fois dans l'histoire de notre République qu'une loi prévoit l'enseignement en langue régionale, et non pas seulement un enseignement des langues régionales. Troisièmement, ces avancées sont accompagnées de dispositifs, comme l'information des familles. Quatrièmement, nous adaptons la loi Deixonne de 1951 en l'étendant aux enseignants du second degré. Cinquièmement, nous reconnaissons la valeur des cultures régionales - votre assemblée a déjà eu un débat sur ce sujet.

\footnotetext{
${ }^{30}$ Voir : https://www.senat.fr/rap/110-657/110-657.html (Rapport nº 657 - 2010-2011 - de Mme Colette MÉLOT, fait au nom de la commission de la culture, de l'éducation et de la communication, déposé le 22 juin 2011).

${ }^{31}$ Voir les débats à l'adresse https://www.senat.fr/cra/s20110630/s20110630 6.html

32 Voir l'amendement ici : http://www.assemblee-nationale.fr/14/amendements/0767/AN/1549.asp; voir la discussion sur http://www.assemblee-nationale.fr/14/cri/2012-2013/20130178.asp\#P503 40537
} 
Bref, intégré au code de l'Éducation, l'article 27 bis devient l'article 40 :

Les langues et cultures régionales appartenant au patrimoine de la France, leur enseignement est favorisé prioritairement dans les régions où elles sont en usage.

Cet enseignement peut être dispensé tout au long de la scolarité selon des modalités définies par voie de convention entre l'État et les collectivités territoriales où ces langues sont en usage.

Le Conseil supérieur de l'Éducation est consulté, conformément aux attributions qui lui sont conférées à l'article L. 231-1, sur les moyens de favoriser l'étude des langues et cultures régionales dans les régions où ces langues sont en usage.

L'enseignement facultatif de langue et culture régionales est proposé dans l'une des deux formes suivantes:

$1^{\circ}$ Un enseignement de la langue et de la culture régionales;

$2^{\circ}$ Un enseignement bilingue en langue française et en langue régionale.

Les familles sont informées des différentes offres d'apprentissage des langues et cultures régionales ${ }^{33}$.

Encore en 2016, au cours du débat avorté ${ }^{34}$ sur la proposition de loi déposée par le député socialiste Bruno Le Roux ${ }^{35}$, c'est au nom de l'article 2 que le chevènementiste ${ }^{36}$ Jean-Paul Laurent refuse toute présence des langues régionales dans l'espace public, école comprise :

La question de la langue est une question politique majeure, qu'il faut aborder avec des principes et une ligne politique. Les principes sont simples : ils sont inscrits dans les articles de la Constitution. Article 2 : «La langue de la République est le français. » Article 75-1 : «Les langues régionales appartiennent au patrimoine de la France. ». La ligne politique doit être déduite de ces principes : rien à l'école, rien dans les services publics, aucune forme de co-officialité, mais un soutien franc aux langues régionales en danger de disparition, par une politique culturelle, nationale et régionale. C'est sur cette base que nous devrions nous rassembler.

Plutôt que cette clarté, les promoteurs des langues régionales - pour reprendre le titre de la proposition de loi - n'ont pour seul souci que de franchir la ligne jaune, de violer les principes, de jouer avec la Constitution.

Et c'est encore et toujours au nom de l'article 2, renforcé par un avis du Conseil d'État, qu'est rejetée, par divers députés de toutes tendances dans tous les débats les plus récents, toute possibilité de dépasser dans le cadre de l'enseignement bilingue public le seuil de la parité absolue (50\% du temps pour le français, $50 \%$ pour la langue régionale).

Il y a donc l'article 2 .

Mais, de façon plus surprenante, il y a aussi la vénérable ordonnance de Villers-Cotterêts (1539), volontiers convoquée par les adversaires de toute avancée pour les langues régionales. C'est le cas de Jean-Paul Laurent dans le même débat :

La langue est un objet sérieux. On répète trop souvent, avec Renan, que la nation est un projet. Or la nation est aussi une langue, une langue commune, une langue vivante. L'ordonnance de Villers-Cotterêts a lancé l'unification linguistique.

\footnotetext{
${ }^{33}$ Loi no $2013-595$ du 8 juillet 2013 - art. 40

34 Lire le compte rendu de ces débats $(30 / 11: 2016)$ sur http://www.assemblee-nationale.fr/14/cri/20162017/20170065.asp\#P921015 et http://www.assemblee-nationale.fr/14/cri/2016-2017/20170066.asp

${ }^{35} \mathrm{http}: / /$ www.assemblee-nationale.fr/14/pdf/propositions/pion4096.pdf

${ }^{36}$ Jean-Pierre Chevènement, socialiste, plusieurs fois ministre, défend un point de vue centraliste, hostile à tout particularisme au sein de la République.
} 
On laisse ici de côté le fait que le texte de Renan, quand on le lit bien ${ }^{37}$, ce qui est rarement le cas de ceux qui l'évoquent, explique justement que la langue ne peut pas servir à fonder la nation...

Tout récemment, le 13 février 2020, un député de la France Insoumise, Bastien Lachaud, lors du débat sur la proposition de loi Molac, a choisi lui aussi de se référer à l'ordonnance de Villers-Cotterêts pour la présenter ni plus ni moins comme un moyen d'assurer, par l'usage du seul français l'unité de tous devant la loi. On admire au passage cet hommage d'un « républicain » à une loi symbolique des vrais débuts de l'absolutisme monarchique, ce qui pose quand même question en termes de cohérence idéologique, et, par ailleurs, de simple vraisemblance historique : indépendamment de leur caractère de sujets de sa majesté - et non de citoyens -, les Français de l'époque étaient certainement plus handicapés face à la justice par le refus de celle-ci d'employer leur langue régionale que par l'imposition dans les lieux de pouvoir de la seule langue française. Et il est assez savoureux de constater que ces défenseurs de l'Ordonnance de 1539, qui se réclament tous deux de la gauche, semblent ignorer que l'Ordonnance en question proscrit aussi les « coalitions ouvrières », ce que l'on appellera plus tard les syndicats, et évoque aussi ce que doit être la procédure de la torture...

Les mêmes ou d'autres peuvent aussi se référer au décret Merlin du 2 thermidor An II $^{38}$ sur l'obligation d'employer le seul français dans les actes publics, si peu révolutionnaires montagnards qu'ils puissent être par ailleurs. Et quand il s'agit de l'enseignement associatif immersif et de la possibilité pour les collectivités locales de le subventionner, c'est la loi Falloux de 1850, pas montagnarde du tout, elle, qui est appelée en renfort.

Cela dit, la question des langues régionales telle que discutée par les parlementaires ne se résume pas à la seule dimension juridique, et, somme toute, les arguties que nous avons évoquées peuvent parfois recouvrir un non-dit proprement idéologique.

\section{La dimension idéologique}

\section{En interne : les « langues du cœur », des « petites patries » et des « cultures régionales »}

Une première remarque : tout le monde, dans tous les débats, s'accorde à saluer la richesse des langues régionales et leur intérêt. Et ce sont de telles manifestations de reconnaissance symbolique qui ouvrent la plupart des interventions sur le sujet, même si certains propos sont complétés par un «mais » plus ou moins affirmé. Au-delà de cet affichage de bons sentiments, s'il y a des «pour », il y a donc aussi, de facto, des «contre », qui sont d'ailleurs ceux qui gagnent à la fin.

Les «pour» se recrutent dans pratiquement tous les partis, sauf le Front /Rassemblement National, quand il a des élus, ou les quelques chevènementistes qui ont survécu à plusieurs alternances.

\footnotetext{
${ }^{37}$ « Les langues sont des formations historiques, qui indiquent peu de choses sur le sang de ceux qui les parlent, et qui, en tout cas, ne sauraient enchainer la liberté humaine quand il s'agit de déterminer la famille avec laquelle on s'unit pour la vie et pour la mort. Cette considération exclusive de la langue a, comme l'attention trop forte donnée à la race, ses dangers, ses inconvénients. Quand on y met de l'exagération, on se renferme dans une culture déterminée, tenue pour nationale ; on se limite, on se claquemure. On quitte le grand air qu'on respire dans le vaste champ de l'humanité pour s'enfermer dans des conventicules de compatriotes. Rien de plus mauvais pour l'esprit ; rien de plus fâcheux pour la civilisation. », Ernest Renan, « Qu'est-ce qu'une nation ? » Conférence en Sorbonne, le 11 mars 1882, disponible en ligne: http://www.iheal.univ-paris3.fr/sites/www.iheal.univparis3.fr/files/Renan___Qu_est-ce_qu_une_Nation.pdf

38 Disponible en ligne à l'adresse : https://www.legifrance.gouv.fr/affichTexte.do?cidTexte= JORFTEXT000000295886\&categorieLien $=\mathrm{id}$
} 
Il s'agit le plus souvent d'élus de territoires concernés par la question : les mieux représentés, les députés ou sénateurs alsaciens, bretons, quelques Occitans, mais aussi (au moins pour une intervention en passant) des «défenseurs » des langues d'oïl, du flamand ou des langues d'immigration.

Dans le cas de l'occitan, certains mettent cependant en doute l'unité de la langue au profit de la reconnaissance de telle ou telle de ses variantes. Ceux-là peuvent partager avec leurs adversaires la formule "langues d'oc», contradictoire de la terminologie reconnue par les ministères de la Culture et de l'Éducation Nationale («occitan» et «langue d'oc » au singulier). Ce qui n'empêche pas la ministre de la culture Christine Albanel de parler de « langues d'oc » dans le débat sans vote du 7 mai 2008 :

Songeons au breton, venu de Grande-Bretagne au $\mathrm{V}^{\mathrm{e}}$ siècle, et qui est la seule langue du groupe celtique encore parlée sur le continent. Songeons aussi au flamand, au francique, à l'alsacien, au corse, à toutes les langues d'oïl et d'oc ${ }^{39}$,

tandis qu'au cours du débat au Sénat le 27 octobre 2015 au sujet de la Charte, Christiane Taubira, garde des Sceaux, oppose tel mot ("amour») qui viendrait des «troubadours provençaux » à tel autre (« cadet »), qui viendrait, lui, de « l'occitan gascon ${ }^{40}$ :

Le français doit, par exemple, l'« amour » aux troubadours provençaux, le «bijou » au breton - la « cohue », aussi ! (Sourires.) -, le « cadet » à l'occitan gascon...

Deux ans plus tôt (22 janvier 2014), le débat sur le même sujet se tenant à l'assemblée avait permis au communiste Chassaigne, favorable au demeurant, de signaler le danger qu'un « occitan niveleur » pouvait faire courir aux variantes réellement pratiquées (écho probable des sollicitations d'un groupe particulariste auvergnat).

Certains se réfèrent à un vécu et affirment que la langue qu'ils défendent est leur langue d'enfance : le centriste Jean Lassalle, qui intervient assez régulièrement tout au long des années étudiées, rappelle à plusieurs reprises que sa première langue a été le béarnais (il n'emploie pas le vocable « occitan »), la seconde l'espagnol (car il accompagnait son père berger en Espagne,) et la troisième le français, appris à l'école.

D'autres incluent dans leur discours de brefs passages (proverbes ou citations d'auteurs) dans leur langue régionale de référence (quitte à être parfois interrompus par des collègues ou par le président de séance qui leur rappellent que la langue normale de l'assemblée est le français). Un exemple, lors de la séance au Sénat du 27 octobre 2015 :

M. Ronan Dantec. Memez, ar brezonegh zo eur yezh flour $!^{41}$ Depuis maintenant plusieurs décennies, cette phrase m'accompagne et nourrit mes interrogations sur la marche du monde, sur ses mutations culturelles, sur le droit redoutable que s'arrogent ceux qui portent l'action publique de décider pour autrui ce qui sera bon pour lui.

Memez, ar brezonegh zo eur yezh flour ! : «Quand même, la langue bretonne est une bien belle langue !» Cette traduction littérale appauvrit un peu le sens du mot flour, plus riche et plus précis que « beau » ou « magnifique ». Dans flour, il y a aussi l'idée de douceur. ${ }^{42}$

On note en passant que la transcription de leurs propos, quand elle est faite, ne respecte pas forcément les règles orthographiques de la langue en question, soit que le transcripteur les

\footnotetext{
${ }^{39} \mathrm{http}: / /$ www.assemblee-nationale.fr/13/cra/2007-2008/153.asp

${ }^{40} \mathrm{https} / / /$ www.senat.fr/seances/s201510/s20151027/s20151027007.html

${ }^{41} \mathrm{Si}$ on adopte le système orthographique -zh, c'est $u r$, non eur et brezhoneg qu'il faut écrire...

${ }^{42}$ http://www.senat.fr/seances/s201510/s20151027/s20151027007.html
} 
ignore, soit, et c'est peut-être la vraie explication, parce que l'orateur lui-même, s'il fournit un texte écrit, n'en sait pas plus ${ }^{43}$.

L'audition des enregistrements vidéo révèle parfois, au demeurant, une maitrise approximative de la langue, comme dans le cas de l'intervention de Jacques Bompard (non inscrit, extrême-droite, Vaucluse) essayant de réciter du Mistral le 30 novembre 2016 (débat sur la proposition Le Roux). Nous avons jugé intéressant de reproduire ici les échanges qui ont eu lieu, tout en renonçant à rétablir la graphie mistralienne, implicitement revendiquée, de la citation $^{44}$ :

M. Jacques Bompard. Madame la présidente, madame la secrétaire d'État, madame la rapporteure, voilà ce que Frédéric Mistral disait à Saint-Rémy-de-Provence, en 1886 [sic pour 1868], devant les poètes catalans :

"Voulèn que nàsti drôle, au-lià d'èstre éleva dins lou mesprés de nosto lengo (ço que fai que, plus tard, mespresaran la terro, la vièio terro maire ounte Dieu lis a fa naisse), voulèn que nàsti drôle countùnion de parla la lengo de la terro, la lengo ounte soun mèstre, la lengo ounte sounfièr, ounte soun fort, ounte soun libre.

Voulèn que nàsti chato, au-lià d'èstre elevado dins lou desden de nàsti causo de Prouvènço, aulià d'ambiciouna li fanfarlucho de Paris o de Madrid, countùnion de parla la lengo de soun brès, la douço lengo de si maire, e que demoron, simplo, dins lou mas ounte nasquèron, e que porton longo-mai lou riban arlaten coume un diadèmo de rèino. "

Mme la présidente. Monsieur le député, permettez-moi de vous interrompre un instant pour vous rappeler que, dans l'hémicycle, vous devez vous exprimer en français.

Mme Annie Genevard. Exactement !

M. Marc Le Fur. Frédéric Mistral fut prix Nobel de littérature!

M. Jacques Bompard. Je vous en donne la traduction, parce que je comprends que vous soyez un peu perturbés : $[\ldots]^{45}$

Je dois bien vous dire que l'enfant de Buis-les-Baronnies que je suis est très fier de vous faire entendre ce beau provençal, même s'il choque certains, au cœur d'une assemblée qui se distingue trop souvent par son centralisme et son jacobinisme. Au début du $\mathrm{XX}^{\mathrm{e}}$ siècle et à la fin du XIX ${ }^{\mathrm{e}}$ siècle, le Félibrige joua d'ailleurs à plein contre ces vieilles traces de radicalisme cherchant à énerver les familles bretonnes, corses ou basques. Je pense que, devant l'échec complet du projet d'arasement de nos singularités locales, il est grand temps de rappeler que la France est composée de peuples et de provinces, dont la richesse n'a d'égale que la diversité.

D'autres se contentent de citer des noms d'auteurs, à commencer par Christiane Taubira, lors de la séance du 27 octobre 2015 au Sénat :

\footnotetext{
${ }^{43}$ Le paradoxe est que, pour acquérir le statut et la considération de langue, la codification est en effet généralement considérée et exigée comme un prérequis par ceux-là mêmes qui lui dénient ce statut. Deuxième paradoxe : le parlementaire défenseur de la langue, parce qu'il manifeste cette incompétence graphique, incarne la diglossie. Aussi parce que cette codification orthographique peut être moins normative que pour le français, voire qu'elle peut être assouplie, comme pour le corse, considéré comme langue polynomique.

${ }^{44} \mathrm{http} / / /$ www.assemblee-nationale.fr/14/cri/2016-2017/20170066.asp\#P921749

45 Traduction de Jacques Bompard lui-même, pour ses collègues députés : "Nous voulons que nos fils, au lieu d'être élevés dans le mépris de notre langue (ce qui fait que, plus tard, ils mépriseront la terre, la vieille terre maternelle sur laquelle Dieu les a fait naitre), nous voulons que nos fils continuent à parler la langue de la terre, la langue dans laquelle ils sont fiers, par laquelle ils sont forts, par laquelle ils sont libres.

Nous voulons que nos filles, au lieu d'être élevées dans le dédain de nos coutumes de Provence, au lieu d'envier les fanfreluches de Paris ou de Madrid, continuent à parler la langue de leur berceau, la douce langue de leurs mères, et qu'elles demeurent, simples, dans les fermes où elles naquirent, et qu'elles portent à jamais le ruban d'Arles comme un diadème de reine. »
} 
Frédéric Mistral a sans doute fait «chavirer » - terme provençal - tous les cœurs, en 1904, lorsqu'il a reçu le prix Nobel de littérature pour une œuvre en langue régionale.

Les auteurs sont nombreux à faire vivre les langues régionales. Je pense à Anjela Duval pour la Bretagne - je vais vous faire voyager ! - à André Weckmann pour l'Alsace, à Jean Aritxelha pour le Pays basque, à Alfred Parépou et Élie Stephenson pour la Guyane, à Monchoachi et Jean Bernabé pour la Martinique, à Hector Poullet et Sylviane Telchid pour la Martinique, à Axel Gauvin et Davy Sicard pour la Réunion, à Nassur Attoumani pour Mayotte, parmi beaucoup d'autres...

C'est ainsi qu'au cours du débat sans vote du 7 mai 2008, l'Antillais socialiste Victorin Lurel cite Aimé Césaire, tandis que l'Aveyronnais UMP Yves Censi cite l'écrivain d'oc Félix Castan ; au cours du débat sur la charte d'octobre 2013, le député de droite Le Fur cite (mais en traduction française) le poète breton Yann-Ber Calloc'h, mort au front pendant la première guerre mondiale.

Ces citations sont parfois maladroites : tel sénateur alsacien peut ainsi, au cours du débat du 27 octobre 2015, se référer à «l'éminent juriste René Schickelé Gesellschaft » : or, si René Schickelé a bien existé mais dans les années 30, il n'était pas juriste mais écrivain. On parle ici d'une association (Gesellschaft) anciennement et honorablement connue en Alsace par ailleurs, qui a pris son nom comme emblème.

Au cours du même débat, le socialiste Georges Labazée évoque, lui, Bernard Manciet, quitte à en faire un auteur «béarnais » ce que ce Landais aurait peu apprécié s'il n'avait pas été déjà mort.

Bruno Retailleau (LR) cite de son côté Giono, Mauriac, Hélias et Mistral. Or, si les deux derniers ont écrit dans une langue régionale (le Breton Hélias au demeurant étant plus connu pour son œuvre en français), les deux autres non seulement n'ont pas écrit en occitan mais pensaient au surplus peu de bien de cette langue : signe d'une confusion fréquente en France où l'on accole l'étiquette régionaliste aussi bien à des auteurs occitans, bretons, alsaciens... qu'à des écrivains de terroir n'écrivant qu'en français.

On note que plusieurs élus socialistes ne manquent pas de citer, plus ou moins correctement, les propos de Jean Jaurès sur les bienfaits du bilinguisme, bienfaits que d'autres, rares au demeurant, peuvent évoquer en termes plus généraux, au nom de l'ouverture à l'altérité : en juillet 2008, un sénateur du Bas-Rhin, Francis Grignon, affirme ainsi

dans un monde en pleine globalisation des échanges et des cultures, nous serons plus performants si nous comprenons l'autre plutôt que si nous l'ignorons. C'est pourquoi grâce à nos langues régionales, nous habituerons nos enfants à cette gymnastique de l'esprit qui leur permettra d'apprendre plus rapidement d'autres langues.

C'est à peu près le même langage que tient la socialiste Frédérique Espagnac au Sénat (27 octobre 2015) même si au passage elle affirme que dans sa circonscription des Pyrénées Atlantiques sont parlées trois langues, le basque, le béarnais et... l'occitan, là encore, selon toute probabilité, pour ne pas froisser d'actifs milieux localistes.

Le vocabulaire employé renvoie souvent à la notion de patrimoine : c'est somme toute normal puisque c'est le terme qui figure dans le seul article de la Constitution mentionnant les langues régionales. Pour la plupart de ceux qui interviennent en faveur de ces langues, on remarque ainsi que c'est souvent au passé plus qu'à la création contemporaine que l'emploi du mot les mène à les associer.

Rares sont ceux qui, comme le sénateur basque Lasserre (27 octobre 2015) signalent qu'il s'agit d'un patrimoine vivant, pas de vieilles pierres, ou qui, comme l'écologiste de Rugy en mai 2008, parlent de patrimoine de l'humanité et de droits de l'homme. Certains vont plus loin et soulignent l'utilité des langues régionales - certaines d'entre elles du moins - dans les 
rapports économiques et commerciaux à l'échelle européenne. Sans surprise on trouve sur ce terrain des élus alsaciens qui valorisent l'importance de l'alsacien pour la recherche d'emplois en Allemagne.

Ceci étant, on n'échappe pas parfois à une certaine folklorisation, liée ou non à la dimension « vécu ».

André Reichert, qui commence sur une note favorable avant de se prononcer finalement contre l'adoption de la Charte, inutile selon lui (octobre 2015, Sénat), donne ainsi à ses collègues (que cela amuse beaucoup, on ignore pourquoi) un cours assez particulier de « dialecte » alsacien :

M. André Reicherdt. ... Souvent appris dans un cadre familial, il est une langue truculente, savoureuse : savez-vous, mes chers collègues, qu'il existe plus de quatre-vingts expressions pour dire des mots doux à une femme? (Sourires.)

M. Philippe Bas, rapporteur. Seulement quatre-vingts ?

M. Lö̈ Hervé. Des exemples, des exemples !

M. André Reichardt. Je suis prêt à donner des cours aux plus assidus d'entre vous... (Nouveaux sourires). Savez-vous qu'il y a plus de quarante expressions pour dire que l'on a marqué un but en football? Cette langue est pleine de subtilités: un même mot peut avoir plusieurs significations. Nous, les Alsaciens, sommes les seuls à la comprendre et sommes les seuls à la parler. C'est la raison pour laquelle nous voulons la garder et la promouvoir ${ }^{46}$.

\section{Le local, le national et le global : langues régionales, patrie et diversité}

On voit parfois invoquée, notamment par les auteurs des propositions discutées, la mise en conformité de la législation française avec ce qui se passe ailleurs, et le lien entre défense des langues régionales et défense à l'international de la diversité des langues, français compris, est parfois établi. Le 29 janvier 2008, le sénateur socialiste François Marc affirme :

Ce qui est en cause c'est la diversité culturelle. Partout où cette question est traitée en Europe depuis une quinzaine d'années, elle est perçue comme source d'avancées démocratiques. Pourquoi ne serait-ce pas le cas aussi en France?

Ce à quoi Jean-Luc Mélenchon répond, superbe, «parce que c'est nous ! ${ }^{47}$.

En janvier 2014, dans son explication de vote sur la charte au nom du groupe centriste UDI, l'orateur Thierry Benoit parle de l'Europe des peuples :

M. Thierry Benoit. Pour une fois que l'Union européenne lance un message fort pour la construction d'une Europe des peuples ! La langue, c'est le dialogue, c'est l'échange, c'est la communication.

M. Jacques Myard. Voilà qui est intéressant !

M. Thierry Benoit. Vous avez évoqué, cher collègue Henri Guaino, une liste de 75 langues parlées sur le territoire métropolitain et d'outre-mer; c'est une richesse. Cette Europe des peuples, nous la voulons.

M. Daniel Fasquelle. À Bruxelles, on parle anglais, maintenant. ${ }^{48}$

tandis que le communiste Chassaigne en appelle de son côté au respect de la diversité linguistique à l'échelle mondiale :

\footnotetext{
${ }^{46} \mathrm{http}: / /$ www.senat.fr/seances/s201510/s20151027/s20151027013.html

${ }^{47} \mathrm{http}: / /$ www.senat.fr/cra/s20080129/s20080129 8.html\#par 863

${ }^{48} \mathrm{http} / / /$ www.assemblee-nationale.fr/14/cri/2013-2014/20140139.asp\#P172394
} 
[...] Pour l'essentiel, vous vous appuyez, monsieur Guaino, sur une approche de la cohésion sociale qui serait figée, comme si ce qui a fait la France, au fil de son histoire, devait être analysé aujourd'hui de la même façon. Je ne vais pas remonter à l'époque médiévale, mais si on s'arrête au XIX ${ }^{\mathrm{e}}$ siècle, l'unification de la langue a été un double instrument, qui a permis avec l'école de la III ${ }^{e}$ République et ses hussards noirs, les instituteurs, de développer l'alphabétisation et...

\section{Jacques Myard. Et le progrès social!}

M. André Chassaigne. ...de donner une culture. L'instrument de la langue unique a été considéré comme un levier indispensable, à tort, je pense, dans ses excès, puisque l'on a, à côté de cela, interdit l'usage de la langue parlée qui était celle de beaucoup de ruraux. [...] Cette situation, qui était celle du XIX ${ }^{\mathrm{e}}$ siècle et de la $\mathrm{III}^{\mathrm{e}}$ République, a évolué. Aujourd'hui, la cohésion sociale s'appuie au contraire sur la diversité. On pourrait développer ce sujet au regard de la mondialisation et de l'exigence d'un retour à une approche plus locale des choses, sans pour autant faire du folklore ou de l'identité réductrice. C'est au contraire une richesse de la France que cette diversité, en particulier la diversité linguistique, car elle s'accompagne d'une diversité culturelle.

La question que l'on peut se poser aujourd'hui est celle-ci : est-ce que la législation, telle qu'elle existe, suffit pour répondre à cette attente ? [...] La première loi, celle du 11 janvier 1951 relative à l'enseignement des langues et dialectes locaux, alors portée par un socialiste, Maurice Deixonne, est-elle suffisante aujourd'hui ? Est-ce que cette loi fondatrice permet de répondre à la diversité culturelle nécessaire et au multilinguisme que nous souhaitons ? [...] Ce qui me semble important, c'est de pouvoir faire sortir les langues de l'espace privé pour aller vers l'espace public. Se limiter à l'espace privé, malgré les avancées qu'ont pu constituer les lois que je viens de citer, ce n'est pas suffisant. Il faut que ces langues prennent davantage de place dans l'espace public, car nous savons très bien que si la langue n'est pas portée dans l'espace public, elle disparaitra. Or pour qu'elle ne disparaisse pas, il faut faire avancer une partie de notre législation et s'appuyer sur cette charte, indispensable aujourd'hui. (Applaudissements sur les bancs des groupes GDR, $S R C$, écologiste et RRDP.)

Est souvent exprimée l'idée qu'il est temps de rendre justice à des langues trop longtemps niées. Christiane Taubira, dans son rôle de garde des Sceaux défendant la ratification de la Charte devant le Sénat en octobre 2015 a cette formule : «l'écrêtement de la diversité culturelle et identitaire conduit à l'uniformisation, et celle-ci à l'exclusion ».

Dès mai 2008, le sénateur de l'Aude Roland Courteau (socialiste) proposait de son côté de « réconcilier la langue de la République et la République des langues ».

Parfois émerge l'idée des blocages sur le terrain, comme dans l'intervention de la socialiste Colette Capdevielle (janvier 2014) dénonçant l'arbitraire de certaines administrations locales, après avoir dénoncé «l'ethnocentrisme jacobin». Ces «jacobins» qui sont d'ailleurs régulièrement étrillés par des élus de tous les partis, certains ajoutant l'ordonnance de VillersCotterêts, dont on a vu le poids qu'elle représentait encore dans l'imaginaire des défenseurs du français seul.

On ne manque pas en revanche d'évoquer le patriotisme des locuteurs des langues régionales, comme ces poilus de 14-18 qui parlaient occitan ou breton, ou les marins bretons de l'ile de Sein partant rejoindre De Gaulle en 1940, voire les fédérés marseillais ou bretons de 1792 qui chantaient un Chant de l'Armée du Rhin dont ils ne pratiquaient pas la langue.

Quant à Aurélie Filipetti, intervenant en qualité de ministre de la Culture dans le débat à l'Assemblée sur la charte de janvier 2014, c'est Marianne, symbole de la République inventé par un révolutionnaire languedocien en 1792 dans une chanson en occitan qu'elle appelle au secours de ceux qui ne pensent pas que les langues régionales menacent la République :

Il va de soi que c'est un avantage pratique d'avoir une langue commune, et le lien de la langue française fonde un sentiment très fort d'appartenance à une même nation, mais ce qui nous unit et nous rassemble, c'est aussi l'adhésion aux droits de l'homme, à la laïcité, au pluralisme, qui 
sont des valeurs éminemment politiques. C'est en français qu'a été proclamée la Déclaration des droits de l'homme, mais c'est en occitan qu'a été créé le personnage de Marianne, symbole de la République, en $1792 .^{49}$

Car somme toute, les arguments développés sont le plus souvent moins agressifs que défensifs. Il est fréquent de voir affirmé que les langues régionales ne menacent pas le français, ou qu'elles ne le menacent plus, sans préciser à quel moment et de quelle façon elles auraient pu le faire. D'autant plus, soulignent certains, que de toute façon ces langues ne sont plus guère parlées, et encore moins transmises en famille, et qu'elles sont donc à la fois menacées et fort peu menaçantes ; le paradoxe sous-jacent étant qu'elles devaient être combattues quand elles montraient une certaine vitalité, et qu'elles doivent désormais être défendues parce qu'elles ne sont plus que moribondes (plutôt comme patrimoine mort que comme patrimoine vivant). C'est ce que dit François Marc, déjà cité, en janvier 2008 :

Dans nos régions, $2 \%$ au plus des enfants apprennent à devenir des locuteurs réguliers de langues régionales. Cela ne risque pas de mettre en danger l'unité de la République !

Des années plus tard, au Sénat en octobre 2015, Christiane Taubira cite même les chiffres tirés de l'enquête INED de 1999 qui confirment cet affaiblissement de la pratique et de la transmission :

En 1910, il existait plus d'un million de locuteurs bretons ; aujourd'hui, ils seraient 250000 . Au début du $\mathrm{XX}^{\mathrm{e}}$ siècle, tous les Alsaciens étaient réputés maitriser l'alsacien, comme les Corses avaient tous la maitrise de la langue corse ; aujourd'hui, sont identifiés 900000 locuteurs alsaciens, sur une population totale de 1,7 million de personnes, et 170000 locuteurs corses pour 250000 insulaires. En ce qui concerne la langue basque, à peine un quart de la population du Pays basque aurait encore aujourd'hui une compétence dans cette langue. Les locuteurs occitans seraient passés de 10 millions en 1920 à 2 millions aujourd'hui. On compterait 110000 locuteurs catalans et 80000 locuteurs flamands, ainsi que 2 millions de créolophones.

Et pratiquement tous les auteurs de propositions de loi, de Navarro à Molac, ne manquent jamais d'assurer que reconnaitre des langues n'aboutit pas à reconnaitre leurs locuteurs comme constituant des « groupes », voire des communautés susceptibles de menacer l'indivisibilité de la Nation : une façon, d'ailleurs vaine, de tenter de désamorcer un des arguments fétiches des adversaires de toute évolution du statut des langues en France.

\section{Une certaine bienveillance, mais...}

Car ces adversaires existent, et, comme les «pour », ils existent dans tous les partis; d'où parfois conflits internes à ces partis qui se révèlent au grand jour : ce sont ceux qui complètent leur hommage obligé aux langues régionales par un « mais » ou un « cependant » ouvrant sur des prises de position radicalement opposées à celles que nous avons vues jusqu'ici. Ils mobilisent plusieurs types d'arguments.

Il y a d'abord l'argument purement pragmatique de ceux qui considèrent que, quel que soit l'intérêt du sujet, ce n'est pas le moment de le traiter et que le débat en cours porte sur autre chose (bien plus important). Répondant à François Marc en janvier 2008, c'est ce qu'indique la garde des Sceaux Rachida Dati : «Les langues régionales ne sont pas l'objet du présent texte. Le gouvernement n'entend d'ailleurs pas rouvrir pour l'instant ce débat ». Ce à quoi Roland Courteau répond «ce n'est jamais le moment », comme déjà Jean Lassalle (Modem), JeanJacques Urvoas (PS) et Michel Vaxès (PCF) confrontés au même propos quelques jours auparavant à l'Assemblée. Dans le meilleur des cas, le ministre annonce que malgré tout, la

\footnotetext{
${ }^{49} \mathrm{http}: / /$ www.assemblee-nationale.fr/14/cri/2013-2014/20140140.asp\#P172609
} 
question sera abordée dans l'hémicycle, mais un autre jour : de fait, à l'issue du débat sans vote du 7 mai suivant, Christine Albanel annonce le dépôt prochain du projet de loi déjà évoqué plus haut et qu'on attend encore.

Autre argument se réclamant d'une certaine bienveillance : ce qui se fait déjà pour les langues régionales, à législation constante (et à moyens proportionnels), est déjà bien suffisant. C'est ce qui ressort par exemple du débat avorté sur la proposition de loi du sénateur Navarro en juin 2011, que ce soit à travers les propos de la rapporteure UMP Colette Melot (il ne faut pas tout attendre de l'État qui fait déjà beaucoup), ou ceux du ministre Chatel, nourris de chiffres, d'où il ressort que le vrai problème c'est que l'action de son ministère n'est pas assez connue. Bref, tout est déjà prévu par les textes (règlementaires) existants, il suffit de savoir les utiliser.

La même argumentation avait été défendue, toujours avec des chiffres, par Christine Albanel en mai 2008, et elle le sera de nouveau le 27 octobre 2015, toujours avec une avalanche de chiffres par la centriste Catherine Morin-Desailly :

Il serait inexact de prétendre que les langues régionales sont aujourd'hui marginalisées ou méprisées, comme je l'ai entendu dire tout à l'heure. Au contraire, soyons objectifs et regardons le verre à demi-plein. Depuis plusieurs années, même si les choses restent perfectibles bien sûr, de nombreux progrès ont été réalisés en matière de diversité culturelle et linguistique.

Ainsi, la loi du 30 septembre 1986 charge les composantes du service public audiovisuel d'assurer la promotion de la langue française et des langues régionales, ainsi que de mettre en valeur la diversité du patrimoine culturel et linguistique de la France.

Le Conseil supérieur de l'audiovisuel, le CSA, a autorisé plus de soixante stations de radio diffusant des émissions en langues régionales.

France Télévisions joue également un rôle essentiel dans la diffusion de programmes en langues régionales. En 2013, près de 585 heures de programmes en langues régionales ont été diffusées sur les antennes de France 3, ainsi que plus de 1100 heures sur la chaine France 3 Corse ViaStella. [...]

Sur le terrain, l'enseignement des langues régionales connait une forte progression, mes chers collègues. En 2012, pas moins de 272000 élèves apprenaient une langue régionale, mobilisant près de trois mille enseignants.

Et d'évoquer, dans la foulée, «l'excellent rapport » de « notre collègue Colette Mélot [...] dès 2011.

C'est le même argument, tout va pour le mieux, que l'on retrouve un peu plus tard (2016), dans l'autre camp, avec Sylvie Grellier, secrétaire d'État aux collectivités, au moment de l'enterrement de la proposition Le Roux.

À ce stade, certains intervenants, généralement d'opposition, désireux de prouver malgré tout leur bienveillance, évoquent alors la question des moyens nécessaires, que le gouvernement ne fournit pas : c'est le sens de l'intervention du communiste Gérard Le Cam le 30 juin 2008 qui tout en combattant la Charte européenne estime que mieux vaudrait une loi, et «surtout militer en faveur de moyens suffisants ».

Le même, dans la foulée, rappelle que « la langue française... elle aussi... mérite toutes les attentions ». Une ligne suivie par bien d'autres orateurs qui entendent bien relativiser l'importance de la question des langues régionales alors même que c'est le français qui est le plus menacé, aussi bien de l'extérieur par la concurrence de l'anglais que de l'intérieur par la montée de l'illettrisme. C'est l'argument du sénateur radical Jacques Mézard le 27 octobre 2015, dans le débat sur la ratification de la Charte ; à sa suite, la centriste Jacqueline Gourault défend, elle, la place du latin et du grec. Catherine Morin-Dessailly, de son côté, affirme, dans le même débat : 
... je ne voudrais pas que ce débat nous fasse oublier d'autres priorités : la maitrise de la langue française, qui est loin d'être acquise pour un grand nombre de nos concitoyens, et celle des langues vivantes étrangères, lesquelles sont toujours plus nécessaires pour l'insertion professionnelle et les échanges internationaux [...]. Faire reculer l'illettrisme est selon moi aujourd'hui la priorité des priorités. Faute de maitriser la langue française, quelque 2,5 millions de nos concitoyens ne sont pas en mesure de faire face aux exigences de la vie quotidienne et de participer à la vie publique. Enfin, j'évoquerai un autre enjeu de taille, mes chers collègues : la pérennité de la francophonie. Dans un monde toujours plus globalisé, le français est lui-même, si nous n'y prenons garde, en passe de devenir une langue régionale. Dans son Discours sur l'universalité de la langue française, Rivarol écrivait: "Tout ce qui n'est pas clair n'est pas français ».

Remarquons que l'oratrice oublie ici, curieusement, de citer l'ensemble du propos de Rivarol d'où il ressort que ce qui n'est pas clair est latin, anglais, allemand, bref tout ce qui n'est pas le français, à savoir les « langues étrangères », sans être pourtant « langue régionale »...

On retrouve invoqué l'illettrisme dans une intervention d'Annie Genevard (Républicains) dans la discussion de la proposition Le Roux. Parfois la question de la surcharge des programmes (qui remonte à la $\mathrm{III}^{\mathrm{e}}$ République...) apparait, notamment dans des interventions musclées de la chevènementiste Marie Françoise Bechtel au cours du même débat ${ }^{50}$.

Autre angle d'attaque : on ne sait pas trop à quelles langues va s'appliquer le texte proposé : c'est là qu'intervient la question des «langues d'oc » opposées à un occitan unifié, ou, le 28 janvier 2008, dans les propos de Jean-Luc Mélenchon la variété des parlers bretons face au langage artificiel des militants, élaboré au surplus par un collaborateur condamné à la fin de la dernière guerre mondiale (Roparz Hémon dont le nom n'est d'ailleurs pas cité) :

Et comment parler de langues régionales sans entrer dans leur définition? Car de quel créole parle-t-on alors qu'il y en a sept ou huit? Quant à la langue bretonne, admirable en bien des points, nous ne saurions la confondre, alors qu'il en existe cinq, toutes respectables, avec le manuel qui en concentre l'apprentissage, et dont l'auteur a, comme l'on sait, été condamné à mort par contumace.

Dans la foulée, Mélenchon reprend une autre antienne bien connue, celle de l'incapacité supposée des langues régionales à épouser certains registres ou à exprimer telle ou telle réalité contemporaine :

Quelles langues régionales donc, et combien ? L'ancien ministre de la formation professionnelle que je suis sait que le vocabulaire technique manque. S'il ne s'agit que d'appeler une fusée fuseï, le jeu n'en vaut pas la chandelli! Et que ceux que je ne traite pas de communautaristes ne me traitent pas de jacobin intransigeant, c'est un pléonasme! (Rires à droite)

Sous l'ironie moyennement légère, c'est bien un mépris brutal du " patois » incapable de dire la modernité (sans que d'ailleurs soit précisé à quel «patois» il est fait allusion) qui s'exprime ici. Mépris qui s'accompagne d'une survalorisation, voire une essentialisation du français :

Le français est une langue de liberté. Imposée par les rois, elle a pourtant facilité la libre circulation des opprimés sur tout le territoire de la République. La langue française est une langue de liberté, qui reconnait la liberté de toutes les langues. (Applaudissements sur plusieurs bancs à gauche et à droite)

\footnotetext{
${ }^{50}$ http://www.assemblee-nationale.fr/14/cri/2016-2017/20170066.asp\#P922313
} 


\title{
Entre péril et mépris
}

Dans certains cas, la discussion dérape à propos des langues «minoritaires » recensées par Bernard Cerquiglini dans son rapport préalable à la ratification de la Charte en 1999 - berbère, romani...- et des langues de l'immigration, l'arabe en premier lieu : c'est la crainte de voir ces langues profiter de la brèche ouverte par les langues régionales que l'on voit exprimée en octobre 2013 dans l'intervention de Marion Maréchal-Le Pen à propos de la Charte (qui mentionne effectivement le berbère et l'arabe dialectal parmi les langues «non-territoriales » du rapport Cerquiglini).

De ce fait, face à ces langues si dangereuses, l'extrême-droite peut éventuellement considérer, fût-ce de façon purement rhétorique, les langues régionales comme un rempart « identitaire », ainsi que le révèlent les propos déjà cités de Jacques Bompart :

\begin{abstract}
Allons plus loin : je dois bien vous avouer que devant l'accroissement des langues des minorités et allogènes sur notre territoire, nous nous trouvons face à un défi identitaire. Ce défi est très simple : voulons-nous que demain un Président provençal puisse chanter Mireille à l'Élysée ou que l'algérien soit la seule langue à laquelle nous laissons cet espoir ? (Exclamations sur les bancs du groupe socialiste, écologiste et républicain)
\end{abstract}

La préoccupation face à l'étranger traverse d'ailleurs le débat de façon plus générale. Au cours du débat du 18 juin 2008, le sénateur socialiste François Marc signale que le Comité des droits économiques, sociaux et culturels des Nations Unies vient d'exprimer sa « préoccupation que l'État français n'avait pas fourni d'efforts suffisants dans la promotion des langues et du patrimoine culturel régional et minoritaire $»$. Ce qui lui vaut sur-le-champ une interruption du rapporteur Gélard «n'importe quoi !» Un peu plus tard, Jean-Luc Mélenchon, à ce moment-là plus ou moins membre encore du même parti (socialiste) que François Marc, " déplore » que « on » c'est-à-dire ce dernier, ait fait référence à un comité de l'ONU, qui n'a rien à dire sur la Constitution française puisqu'il ne reconnait pas le principe de laïcité (on a peine à voir, soit dit en passant, ce que la laïcité vient faire dans cette affaire).

Propos immédiatement repris par le sénateur de droite Philippe Dallier dénonçant le « comité Théodule du grand machin new-yorkais probablement rédigé dans quelque volapük intégré » (références codées à des propos, jadis, du Général De Gaulle, cité d'ailleurs immédiatement après). Dès le débat sans vote du 7 mai précédent, au demeurant, le sénateur centriste Hunault avait de son côté souligné le danger d'une Charte européenne qui donnait pouvoir à des experts européens de venir évaluer la façon dont la France appliquerait, ou non, la Charte. Cet argument apparait rarement dans les débats sur la Charte, mais il n'est pas impossible qu'il y ait là en fait la vraie raison de l'opposition d'une bonne partie des élus à la ratification.

Lors des débats plus directement politiques, voire à prétention philosophique, comme dans les discussions en apparence purement juridiques autour de la Constitution et de la Charte, ce qui est central, c'est une certaine idée de la France une et indivisible, dont la cohésion pourrait être mise à mal par l'irruption des communautarismes dans le corpus législatif et constitutionnel. C'est alors que les deux premiers articles de la Constitution sont évoqués, au nom de l'unicité du peuple français et de la prééminence du français depuis l'Ordonnance de Villers-Cotterêts de 1539 dont on a vu l'étrange affection qu'elle inspirait à certains chevènementistes ou mélenchonistes, suivis par un communiste (démissionnaire en 2011), le sénateur Ivan Renar, lors des débats du Sénat du 18 juin 2008. Le thème est omniprésent dans les déclarations des adversaires de toute avancée sur la Charte européenne, ce qui amène, on l'a dit, les partisans de celle-ci à multiplier les professions de foi unitaristes. L'expression la plus pure de ce topos se trouve en janvier 2008 dans un des interventions devant le Sénat de Jean-Luc Mélenchon, encore membre du PS à cette date :

Il y a des Français qui, par conviction philosophique, sont fondamentalement opposés à l'idée de doter des groupes de locuteurs de droits particuliers. Ce ne sont pas des jacobins dogmatiques 
[réponse à un autre topos venu " d'en face »] mais tout simplement des républicains. La France n'est pas essentialiste. Elle n'est pas la conjugaison de diversités. Elle est la communauté légale une et indivisible qui fait qu'entre l'État et la personne, il n'y a pas d'intermédiaire. ${ }^{51}$

En dehors du fait que cela sonne bel et bien comme une définition essentialiste, on peut se demander si, à ce titre, ce n'est pas l'ensemble des partis, syndicats et associations qui devraient être dissous, dans la mesure où chacune de ces entités prétend bel et bien incarner les intérêts d'un groupe distinct du reste de la «communauté nationale». Nul ne répond (ou n'ose répondre) à Jean-Luc Mélenchon sur ces points, et il peut donc conclure son intervention, sans surprise, en signalant que somme toute ce qui se fait déjà pour les langues régionales est bien suffisant, et que de toute façon on ne sait pas trop de quelles langues on parle (et ici ressurgit le cas du breton et de sa forme standard élaborée par le collaborateur Roparz Hémon).

Il y a encore autre chose que révèle l'analyse du contenu des discours des adversaires de toute amélioration du statut des langues régionales, quelque chose qui a moins à voir avec les grands principes philosophiques qu'avec ce qui ne peut être qualifié autrement que par le terme de mépris - on l'a entrevu plus haut. Le ton est donné dès le début de la discussion sur le texte de «modernisation des institutions de la $\mathrm{V}^{\mathrm{e}}$ République » et l'insertion d'une mention des langues régionales dans l'article 1 de la Constitution (18 juin 2008).

Le premier orateur à s'exprimer est le sénateur socialiste Michel Charasse, qui se dit

surpris... de voir apparaitre à l'Assemblée nationale un amendement tendant à classer les langues régionales au patrimoine de la France. Pourquoi ne pas y classer aussi les monuments historiques ou même la gastronomie, que certains verraient de surcroit au patrimoine de l'humanité ? Voir la potée auvergnate ainsi distinguée, je n'osais en rêver. [" sourires » note la didascalie qui suit]. ${ }^{52}$

Le rapporteur, Patrice Gélard (droite), renchérit en ajoutant «la franc-maçonnerie, le christianisme et les cathédrales », d'autres, plus loin dans le débat, rajoutent le champagne...

Au cours de la discussion de la proposition Navarro au Sénat, la centriste Catherine MorinDesailly lance «je n'ai jamais entendu de plainte au sujet d'un quelconque déficit d'enseignement du cauchois dans ma région » : on sait en effet que le cauchois, variante du normand, ne fait pas partie des langues régionales enseignées en primaire ou dans le secondaire... La même, après avoir signalé que l'enseignement des langues régionales coute cher, appelle un peu plus loin à faire la différence entre ces langues et les «langues fondamentales »: " il est sans doute plus utile pour un jeune de parler espagnol, anglais, allemand ou chinois qu'un patois qu'il ne pourra utiliser que très localement ». Le cauchois est de nouveau convoqué par la même oratrice le 27 octobre 2015 : " Vous conviendrez en effet que l'on ne peut pas mettre sur le même plan le créole, langue vivante, et le cauchois, qui n'est plus guère utilisé dans ma Normandie natale ».

Au cours de la discussion de la loi Peillon à l'assemblée le 15 mars $2013^{53}$, le rapporteur socialiste Yves Durand déclare d'emblée préférer «l'universel » à «l'ancrage dans le local » tandis que la députée chevènementiste Bechtel associe clairement la défense des langues régionales au « régionalisme conservateur de nos provinces » et à la « ruralité ».

On la retrouve trois ans plus tard, avec le même argumentaire, dans la discussion de la proposition Le Roux (30 novembre 2016). Entre temps, Henri Guaino, intervenant au nom de la droite au cours du débat sur la ratification de la charte (octobre 2013) parle des «vieilles langues de nos provinces » dans un développement où défilent le péril "ethniciste », "l'exception française » héritée de la Monarchie comme de la Révolution, et l'inexistence d'une frontière entre langues d'oïl et d'oc. Lorsque le même débat a lieu au Sénat le 27 octobre

\footnotetext{
${ }^{51} \mathrm{http}: / /$ www.senat.fr/cra/s20080129/s20080129 8.html\#par 863

${ }^{52} \mathrm{https} / / / \mathrm{www} . \mathrm{sen}$ at.fr/seances/s201106/s20110630/s20110630016.html

${ }^{53} \mathrm{http}: / / \mathrm{www}$. assemblee-nationale.fr/14/cri/2012-2013/20130178.asp
} 
2015, le radical cantalien Jacques Mézard (membre donc de la majorité à cette date) ne rend hommage aux «traditions, sonorités de langage en harmonie avec les territoires » que pour mieux refuser de leur laisser place dans le texte sacré de la Constitution.

Le point ultime dans ce registre nous parait atteint par un autre sénateur radical, Jean-Pierre Plancade (Haute Garonne), au cours du débat sur la proposition Navarro (30 juin 2011). Ce sénateur commence son intervention en occitan ou du moins dans un idiome un peu approximatif qu'il présente comme tel :

mousou lo president, mousou lo ministre, madama la raportaïra, cars coleguas, me fa plaser de parlar la lengua de mon enfança mas coma gä̈rebé digus compren vau fau la redirado ${ }^{54}$

Et d'enchainer « Rassurez-vous, je m'en tiendrai là pour l'occitan »; « Nous aimons autant en effet » commente le sénateur de droite Philippe Dallier (« sourires »).

On attendrait là un exposé ému et nourri de vécu sur la nécessité de rendre justice à des langues injustement décriées. De fait, l'orateur évoque le temps où on le punissait à l'école pour avoir parlé «patois ». Mais c'est pour mieux dénoncer «la volonté excessive de rendre l'enseignement d'une langue régionale obligatoire ». Dénoncer aussi le «culte des racines» qui ne peut déboucher que sur «le repli sur soi », le « retour à la terre », le «patriotisme de terroir » sur fond de «conservation nostalgique ». Et de signaler que si en son temps il a pu aider localement à l'enseignement de l'occitan, il préfère que sa petite-fille apprenne le chinois.

Nous avons là une assez extraordinaire illustration de ce que les sociolinguistes occitans et catalans appellent l'auto-òdi, la « haine de soi », ou auto-dénigrement ${ }^{55}$.

On a noté au passage cette idée qu'ouvrir la porte aux langues régionales à l'école ne peut aboutir qu'à leur enseignement obligatoire, disposition qui ne figure pourtant dans aucun des textes examinés, y compris dans les 39 articles de la Charte que la France a signés en 1999. D'autres évoquent le risque de voir le facteur breton muté en Gironde contraint d'apprendre l'idiome local. Ce genre d'arguments qui revient souvent dans les propos d'autres élus de tous les partis, malgré les dénégations de leurs adversaires, témoigne d'une véritable ignorance du sujet et des textes concernés, que cette ignorance soit réelle ou feinte. Signe à tout le moins que le sujet ne parait pas mériter un vrai débat nourri de connaissances solides.

\section{Un spectacle tragi-comique sur toile de fond catastrophique...}

D'autant plus qu'il faut faire intervenir ici une dernière dimension, ce que l'on pourrait appeler le théâtre parlementaire tel qu'on le connait depuis les $\mathrm{III}^{\mathrm{e}}$ et $\mathrm{IV}^{\mathrm{e}}$ Républiques : les interruptions goguenardes qui permettent à tel élu d'apparaitre fugitivement dans le compte rendu d'un débat auquel il ne participe pas vraiment, les private jokes entre vieux complices de bords différents, ou, plus sérieusement, les attaques entre partis rivaux.

C'est Aurélie Filipetti lançant à Henri Guaino au terme de sa diatribe contre la Charte (octobre 2013) « vous semblez penser que l'homme régional n'est pas entré dans l'histoire, mais c'est pourtant le cas »: allusion transparente à une phrase glissée par Guaino dans le discours de Dakar du président Sarkozy et qui lui est depuis régulièrement rappelée sans charité excessive.

Plus sérieusement, au moment de la discussion de la proposition Le Roux, le député républicain Marc Le Fur se fait un plaisir de souligner que, proposer un tel texte 90 jours avant

\footnotetext{
${ }^{54}$ [Monsieur le président, monsieur le ministre, madame la rapporteure, chers collègues, cela me fait plaisir de parler la langue de mon enfance mais comme presque personne ne comprend je vous fais la traduction.] Là encore, nous renonçons à proposer une version orthographiquement normée et nous renvoyons à ce que nous disons plus haut de la difficulté de noter correctement les langues régionales quand elles affleurent dans les débats parlementaires

${ }_{55}$ Rafael Lluís Ninyoles, Conflicte lingüistic valencià, Barcelona, Edicions 62, 1969 ; Carmen Alén Garabato, Romain Colonna (dir.), Auto-odi. La "haine de soi" en sociolinguistique, Paris, L'Harmattan, 2016.
} 
la fin d'une session débouchant sur la fin de la législature, signifie qu'on ne suppose pas que le texte fera l'objet d'une navette parlementaire, bref, que le gouvernement qui lui ouvre l'ordre du jour, alors qu'en cinq ans il n'a déposé aucun projet de loi sur la question, prouve bien qu'il n'y croit pas. Marc Le Fur étant un des vrais spécialistes du sujet, qui intervient dans pratiquement tous les débats à l'Assemblée depuis 2008, il peut se payer le luxe de dénoncer un vrai "gâchis ». À vrai dire, son verdict ne risque pas d'être contredit par le fait que la rapporteure de la proposition donne parfois l'impression de ne pas comprendre elle-même le texte dont elle parle, et que la secrétaire d'État présente en séance oppose à pratiquement tous les articles d'une proposition, pourtant déposée par des élus de son propre parti, un avis défavorable du gouvernement. De fait, d'une législature à l'autre, il se trouve toujours un député ou un sénateur pour demander au parti adverse pourquoi il n'a rien fait pour les langues régionales quand il était aux responsabilités. Ici on dépasse le stade du seul théâtre parlementaire pour toucher à ce qui pourrait bien être l'essentiel.

Précisons : au fond, si la question est si importante, pourquoi ne pas l'avoir traitée avant? $\mathrm{Ou}$ : pourquoi présenter une proposition et non un projet de loi porté par le gouvernement? $\mathrm{Ou}$ : pourquoi la déposer alors qu'on sait que la navette ne pourra pas avoir lieu, ou, variante à propos de la ratification de la Charte, pourquoi attendre octobre 2015 pour soumettre au Sénat une proposition de ratification de la Charte européenne adoptée par l'Assemblée en janvier 2014, alors même qu'on vient de perdre la majorité dans la haute assemblée ? Ou, pour nous tourner cette fois non plus du côté du gouvernement, maitre de l'ordre du jour, mais du côté des partis et des élus, pourquoi inclure dans une proposition de loi des dispositions (sur le financement des écoles associatives ou sur l'extension du bilinguisme au-delà du seuil de la parité) dont on sait d'avance qu'elles seront rejetées ? Tout cela, au risque de mener les observateurs à considérer qu'il ne s'agit que d'une opération politicienne destinée à satisfaire des lobbies « régionalistes » ou « communautaristes » avant telle ou telle élection. L'octroi de l'article 75-1 en 2008 après des mois de débat récurrent sur les langues régionales parasitant un débat plus général peut de ce point de vue apparaitre comme une concession pour solde de tout compte, puisqu'on va vite comprendre que cet article ne sert à rien.

En fait, on voit bien que la question gêne les décideurs que sont censés être les élus : ne serait-ce que parce qu'il y a des partisans et des adversaires des langues régionales dans tous les partis, obligés donc de laisser la liberté de vote à leurs membres, ce qui n'arrive pratiquement jamais dans les débats ordinaires, où chaque groupe vote en bloc, et à laisser faire en leur nom des interventions qui se contredisent.

Plus profondément : ce que révèlent les débats, c'est que, quand tel texte est déposé par tel parti, les autres partis se doivent de manifester leur opposition, quels que soient les sentiments de leurs membres. D'où l'impossibilité de déposer un projet commun, malgré l'existence d'un « intergroupe » à l'assemblée consacré à la question : cet intergroupe élabore en 2010 une proposition de loi transpartisane, portée au départ par le socialiste Urvoas et l'UMP Le Fur. La question se complique au moment de savoir quel nom figurera en tête du texte déposé. On finit par s'entendre sur le nom du président de l'intergroupe, le socialiste Jung. Sur ces entrefaites surgit le 10 novembre un texte distinct déposé par un député UMP, Lecou, ce qui amène Urvoas à dénoncer une manœuvre de la droite, et aboutit à la rupture avec Le Fur une semaine plus tard. Du coup, aucun des deux textes ne sera adopté, ni même discuté vraiment.

$\mathrm{Au}$ fond, la question n'est pas jugée assez fondamentale pour être vraiment traitée (peutêtre, sans doute même, faute d'une vraie pression en société). Aucun parti n'en fait un sujet central dans sa propre stratégie et dans son projet global, mais chacun peut, à l'occasion, s'en servir contre les autres au cours d'une joute verbale plus ou moins spectaculaire, mais de toute façon sans résultat tangible. Signe supplémentaire : aucun gouvernement en place ne se sent obligé de déposer par exemple un projet de loi, même après avoir explicitement promis de le 
faire, comme en 2008 avec Christine Albanel. Dans le pire des cas, comme pour la proposition Le Roux, un gouvernement peut carrément saboter une initiative venant de son propre parti.

Et ce qui frappe, dans les débats en séance mais plus encore dans les (assez inexistants le plus souvent) " exposés des motifs », c'est l'absence de vraie réflexion sur la place des langues régionales dans la culture nationale, au-delà des éléments de langage ordinaires (c'est un patrimoine, il faut les défendre, etc.). Absence de réflexion liée à une ignorance profonde du sujet, extérieur en tout état de cause à la formation que les acteurs ont pu recevoir dans le système éducatif ordinaire et a fortiori dans les grandes écoles qui leur fournissent l'essentiel de leurs outils d'interprétation des problèmes. Du coup, quand le débat s'engage vraiment, on le voit rapidement clos par des considérations répétitives et fétichistes sur les articles 1 et 2 de la Constitution : la société française est une et indivisible, et il n'est pas question de reconnaitre qu'elle est traversée de contradictions. Son pluralisme doit donc être reconnu en théorie - aussi bien « en interne » qu'à l'international -, mais en pratique, combattu, sur le terrain y compris législatif. Sauf que le pluralisme linguistique est bien moins porteur de conflits que d'autres pluralismes (sociaux, politiques, religieux...) comme le démontre surabondamment l'histoire de France. De ce point de vue, la sacralisation de la langue française comme fondatrice de l'identité nationale apparait contradictoire avec la conception de la Nation politique revendiquée par les uns et les autres. Mais qui, parmi les professionnels de la politique en France, se soucie d'identifier cette contradiction? On peut donc s'attendre à devoir dans l'avenir analyser d'autres débats qui, selon toute vraisemblance, ne complèteront qu'à la marge le corpus constitué par les débats qui les ont précédés, et déboucheront sur la même absence de résultats concrets pour les langues de France dont le dépérissement se poursuit, quant à lui, assez inexorablement. 


\section{GLOTTOPOL}

Revue de sociolinguistique en ligne

Comité de rédaction : Michaël Abecassis, Salih Akin, Sophie Babault, Claude Caitucoli, Véronique Castellotti, Régine Delamotte, Robert Fournier, Stéphanie Galligani, Emmanuelle Huver, Normand Labrie, Foued Laroussi, Benoit Leblanc, Fabienne Leconte, Gudrun Ledegen, Danièle Moore, Clara Mortamet, Alioune Ndao, Isabelle Pierozak, Gisèle Prignitz.

Rédactrice en chef : Clara Mortamet.

Comité scientifique : Claudine Bavoux, Michel Beniamino, Jacqueline Billiez, Philippe Blanchet, Pierre Bouchard, Ahmed Boukous, Pierre Dumont, Jean-Michel Eloy, Françoise Gadet, Monica Heller, Caroline Juilliard, Jean-Marie Klinkenberg, Jean Le Dû $(\dagger)$, Marinette Matthey, Jacques Maurais, Marie-Louise Moreau, Robert Nicolaï, Didier de Robillard, Paul Siblot, Claude Truchot, Daniel Véronique.

\section{Comité de lecture pour ce numéro :}

Salih Akin, Carmen Alén Garabato, Sophie Babault, Philippe Blanchet, Henri Boyer, Véronique Castellotti, Marisa Cavalli, Jean-François De Pietro, Didier de Robillard, Alain Di Meglio, Ksenija Djordjevic, Jean Michel Eloy, Pascale Erahrt, Véronique Fillol, Monica Heller, Robert Fournier, Normand Labrie, Hervé Lieutard, Jean Le Dû (†), Marinette Matthey.

http://glottopol.univ-rouen.fr

ISSN : 1769-7425 